testing with 16/18 genotyping and triage with p16/Ki-67 immunocytochemistry.

Methodology Women between 30 and 60 years who had in 12 collaborating centres regular annual Pap smear were cotested in 3 years interval for HPV DNA with selective 16/18 genotyping (Cobas 4800, Roche). All HPV 16/18 positive cases and/or those with severe abnormality in cytology were directly refered to colposcopy; HPV non-16/18 positive cases and LSILs were triaged using p16/Ki-67 dual-stained cytology (CINtec Plus, Roche) and positive cases were refered to colposcopy.

Result(s)* Altogether 2407 patiens were eligible for analysis. Mean age of subjects was 43 years. The first round showed 8 cases with severe and 105 cases with mild Pap smear abnormalities. There were 7.4\% (180/2418) patients with HPV positivity, out of them 50 had HPV 16 and/or 18. Triage using p16/Ki-67 was positive in $22.5 \%$ cases (29/129). After 2 years of follow-up biopsy confirmed 38 HSILs and 2 glandular lesions, all of them were HPV positive.

Conclusion* Screening based on HPV testing with selective 16/18 genotyping and $16 / \mathrm{Ki}-67$ triage proved during three years four times more high-grade lesions including glandular lesions than standard screening based on Pap smears.

\section{PRIMARY PREVENTION OF OVARIAN CANCER: A PATIENTS DECISION AID FOR OPPORTUNISTIC SALPINGECTOMY}

${ }^{1} \mathrm{M}$ Gelderblom*, ' $\mathrm{L}$ Van Lieshout, ${ }^{1} \mathrm{~J}$ De Hullu, ${ }^{2} \mathrm{R}$ The, ${ }^{3} \mathrm{~A}$ Van Ginkel, ${ }^{4} \mathrm{~A}$ Oerlemans, ${ }^{5} \mathrm{~K}$ Smeets, ${ }^{6} \mathrm{M}$ Schreurs, ${ }^{7} \mathrm{~J}$ Piek, ${ }^{4} \mathrm{R}$ Hermens. ${ }^{1}$ Radboud University Medical Center, Department of Obstetrics and Gynecology, Nijmegen, Netherlands; 'ZorgkeuzeLab, Development and Implementation of Decision Aids, Delft, Netherlands; ${ }^{3}$ Rijnstate Hospital, Department of Obstetrics and Gynecology, Arnhem, Netherlands; ${ }^{4}$ Radboud University Medical Centre, Department of IQ healthcare, Nijmegen, Netherlands; ${ }^{5}$ Slingeland Hospital, Department of Obstetrics and Gynecology, Doetinchem, Netherlands; ${ }^{6}$ Medisch Spectrum Twente, Department of Obstetrics and Gynecology, Enschede, Netherlands; ${ }^{7}$ Catharina Hospital, and Catharina Cancer Institute, Department of Obstetrics and Gynecology, Eindhoven, Netherlands

\subsection{6/ijgc-2021-ESG0.545}

Introduction/Background* The discovery of the Fallopian tube epithelium as origin of high grade serous ovarian cancer has brought a new option for ovarian cancer prevention, the opportunistic salpingectomy (OS). The popularity of OS is increasing globally, however at present there is substantial practice variation. As a result, whether or not a woman is able to make her own decision on OS depends on the hospital or gynaecologist she visits. To lower practice variation, we developed and tested a patient decision aid (PtDA) for OS in women undergoing either pelvic gynaecological surgery with the intention to retain the ovaries or a sterilization.

Methodology We followed a systematic development process based on the International Patient Choice Aid Standard (IPDAS). Data were collected between June 2019 and June 2020, using both qualitative and quantitative methods. The development process took place in collaboration with patients and healthcare professionals, was overseen by a multidisciplinary steering group, and was divided in four phases; 1. Assessment of decisional needs using individual interviews and questionnaires; 2. Development of content and format based on decisional needs, current literature and guidelines; 3 . Alpha-testing and first revision round; and 4. Alpha-testing and second revision round.

Result(s)* An outline of the PtDA was developed based on decisional needs, current literature and guidelines. It became clear that the PtDA should consist of two separate paths: one on salpingectomy in addition to abdominal surgery and one on salpingectomy as a sterilization method. Both paths contain information on the anatomy and function of ovaries and Fallopian tubes, the estimated risk reduction of ovarian cancer and the potential benefits and risks of OS. Adjustments were made following alpha-testing round one. The improved PtDA was subjected to usability tests (alpha-testing round two), in which it scored an 'excellent' in patient testing and a 'good' in tests with gynaecologists.

Conclusion* In collaboration with patients and healthcare professionals, a PtDA was developed on OS. Both patients and gynaecologists thought it a usable aid which supports patients in making an informed decision whether to undergo an opportunistic salpingectomy, and supports the counselling process by gynaecologists.

\section{RISK REDUCTION SALPINGO-OOPHORECTOMY IN BRCA MUTATION CARRIERS. PRESURGICAL AND PATHOLOGY FINDINGS. A PROSPECTIVE COHORT STUDY}

${ }^{1} \mathrm{MDLR}$ Oliver*, ${ }^{2} \mathrm{~S}$ Aragon-Sanchez, ${ }^{3} \mathrm{~L}$ Alvaro, ${ }^{4} \mathrm{M}$ De Miguel-Reyes, ${ }^{5} \mathrm{E}$ Felipe-Pardo, 5J Montero, ${ }^{6} \mathrm{M}$ Martinez-Lopez, ${ }^{1} \mathrm{C}$ Álvarez-Conejo, ${ }^{1} \mathrm{G}$ López-Gonzalez, ${ }^{1} \mathrm{~B}$ Gil-lbañez, ${ }^{1} \mathrm{JM}$ Seoane-Ruiz, ${ }^{1}$ A Tejerizo-García. ${ }^{1}$ Hospital Universitario 12 de Octubre, Gynecology Oncology, Spain; ${ }^{2}$ Hospital Universitario 12 de Octubre, Breast Cancer, madrid, Spain; ${ }^{3}$ Hospital Universitario 12 de Octubre, Breast Cancer, Spain; ${ }^{4}$ Hospital Universitario 12 de Octubre, Genetics, Spain; ${ }^{5}$ Hospital Universitario 12 de Octubre, Spain; ${ }^{6}$ Hospital Universitario 12 de Octubre, Pathology, Spain

\subsection{6/ijgc-2021-ESG0.546}

Introduction/Background* Women with germline mutations in the BRCA $1 / 2$ genes have a lifetime increased risk of ovarian cancer, $36-63 \%$ and 10-27\% respectively. Accordingly, once childbearing is completed, Risk Reduction Salpingo-oophorectomy (RRSO) is recommended in this group of patients. The purpose of this study was to determine the presurgical findings and the incidence of Serous Tubal intraepithelial carcinoma (STIC) and occult carcinomas in BRCA mutations carriers in whom a RRSO was performed.

Methodology Prospective study that included patients with documented BRCA mutation who accepted RRSO between January 2011 to January 2021 at the Hospital Universitario 12 de Octubre. The study was approved by the ethics committee of the institution. During the month prior to surgery, a systematic ultrasound (US) and determination of serum $\mathrm{Ca}$ 125 levels were performed. Specialized gynecologists performed RRSO by laparoscopy. Unilateral or bilateral adnexectomy was performed according to the surgical history of each patient. Pelvic washing was done in all cases at the beginning of the procedure and tubes were removed at the uterine insertion. All the histologic exams were performed by pathologists subspecialized in Gynecologic Oncology and the sectioning and extensively examining of the fimbriated end protocol (SEE-FIM protocol) was applied. STIC was defined using a combination of morphologic evaluations to 